\title{
Panorama da inteligência competitiva no Brasil: os pesquisadores e a produção científica na plataforma
}

Lattes

\section{Roniberto Morato Amaral}

Doutor em Engenharia de Produção. Professor Departamento de Ciência da Informação da Universidade Federal de São Carlos

Aline Grasiele Cardoso Brito,

Doutoranda do Programa de Pós Graduação em Ciência, Tecnologia e Sociedade UFSCar. Mestre em Engenharia de Produção EESC/USP

karin Gomes da Silva Rocha

Mestranda em Ciência, Tecnologia e SociedadeBacharel em Biblioteconomia da Universidade Federal de São Carlos

Luc Marie Quoniam,

$$
\begin{aligned}
& \text { Professor titular - Aix Marseille Université, } \\
& \text { Institut de Recherche en Sciences de } \\
& \text { l'Information et de Communication- França }
\end{aligned}
$$

Leandro Innocentini Lopes de Faria

Doutor em Ciência da Informação. Professor Departamento de Ciência da Informação da Universidade Federal de São Carlos

http://dx.doi.org/10.1590/1981-5344/2687

Com base nos desafios presentes e futuros e na importância da consolidação da atividade de Inteligência Competitiva (IC) no Brasil, este artigo teve como objetivo geral identificar e analisar o perfil do pesquisador de IC, quanto à formação, atuação e produção científica no Brasil. O método de pesquisa utilizado compreendeu as técnicas de análise bibliométrica e de conteúdo. A amostra foi composta por 1.434 currículos, dados referenciais de 223 artigos e 26 projetos de pesquisa em andamento, recuperados de forma automatizada na Plataforma Lattes, com a expressão de busca "inteligência competitiva". Os resultados alcançados foram: 1] 
representação visual do perfil de atuação e formação do pesquisador brasileiro em IC; 2] categorização da produção científica e dos projetos de pesquisa em IC; e 3] correlação das temáticas investigadas nas publicações em IC. Conclui-se que os resultados deste artigo, contribuem para o entendimento da interdisciplinaridade da IC e sua legitimidade como campo de pesquisa em Ciência da Informação, ao externalizar o perfil dos pesquisadores envolvidos na investigação científica da atividade de IC.

Palavras-chave: Inteligência competitiva; Pesquisador em IC; Plataforma Lattes; Produção científica.

\section{Competitive intelligence in panorama of brazil: the researchers and scientific production on Lattes platform}

Based on the present and future challenges and the importance of consolidation of Competitive Intelligence activity (CI) in Brazil, this article aimed to identify and analyze the CI researcher profile, the training, expertise and scientific production in Brazil. The research method understood the techniques of bibliometric and content analysis. The sample consisted of 1,434 resumes, reference data of 223 articles and 26 research projects in progress, recovered in an automated manner in the Lattes Platform, with the search term "competitive intelligence". The results were: 1] visual representation of performance profile and formation of the Brazilian researcher in CI; 2] categorization of scientific production and research projects in CI; and 3] investigated the correlation of thematic publications in CI. We conclude that the results of this paper, contribute to the understanding of the interdisciplinarity of CI and its legitimacy as a research field in Information Science, to externalize the profile of the researchers involved in scientific research of IC activity.

Keywords: Competitive intelligence; CI researcher; Lattes Platform; Scientific production.

Recebido 25.05.2015 Aceito em 26.07.2016 


\section{Introdução}

A forte competição entre organizações e nações apresenta continuamente desafios de diferentes naturezas e magnitude, para superá-los, visando alcançar uma vantagem competitiva sustentável (HAMEL; PRAHALAD, 1990), se faz necessário solucionar problemas complexos envolvendo significativo número de atores e volumes de informações. Nesse contexto a Inteligência Competitiva (IC) torna-se uma significativa opção metodológica e prática para o aproveitamento de oportunidades e neutralização de ameaças, mediante a coleta e análise sistemática de informações para o apoio à tomada de decisão (FULD, 1995; SCIP, 2015).

A complexidade das inter-relações das organizações com o seu ambiente econômico, social, político e tecnológico (PORTER, 1989) representa um grande desafio para a prática de IC (FLEISHER; BENSOUSSAN, 2002). Soma-se a isso a dinâmica do processo de produção de inteligência, em torno de um ciclo de várias fases (CALOF, 1999; BATTAGLIA, 1999; MILLER, 2000; BOUTHILLIER; SHEREAR, 2003; NIT, 2004), cujos procedimentos requerem múltiplas competências dos profissionais (PRESCOTT, 2009; AMARAL et al., 2011) e a iteração e interação entre as fases do ciclo, o que amplia o grau de complexidade e de dificuldade para a realização da atividade de IC (NIT, 2004). Todos esses condicionantes recaem sobre os integrantes das equipes atuantes em IC, que precisam ser hábeis na manipulação e combinação de múltiplos métodos e técnicas de coleta e análise de informações sobre a organização e seu ambiente (LAHEY, 2003). O domínio das técnicas pertinentes à IC exige tempo e esforço consideráveis e os profissionais atuantes nessa área devem estar em contínuo processo de aprendizagem (FULD, 1995).

A atividade de IC apoia os processos de tomada de decisão e planejamento estratégico das organizações brasileiras ao providenciar insights sobre qual direção seguir no ambiente competitivo (TARAPANOFF, 2001; GOMES; BRAGA, 2006a). Embora a IC não constitua novidade nos países desenvolvidos, no Brasil, sua investigação científica pela área do conhecimento da Ciência da Informação é reconhecida a partir do ano 2000 como um tema emergente (MUELLER; MIRANDA; SUAIDEN, 2000; SILVA et. al, 2006;). Isto se justifica pelo fato da IC ter como objeto de pesquisa a informação no processo de tomada de decisão permitindo a otimização da estratégia da organização (CAPUANO et al., 2009; PINHEIRO, 2006; MARCIAL; SUAIDEN, 2016).

$\mathrm{Na}$ Ciência da Informação e em áreas correlatas aspectos relacionados à profissionalização, formação, funções e às competências de equipes de IC foram discutidos por autores como Fuld (1995), Calof (1999), Vargas e Souza (2001), Miller (2000), Fleisher, (2003; 2004), Herring (2006), Lahey (2003), Dilworth (2003) e Prescott (2009). Em especial no Brasil é possível identificar estudos que versam sobre a caracterização da produção científica em IC (PERUCCHI; ARAUJO JUNIOR, 
2012), a estrutura científica da IC (MARCIAL; SUAIDEN, 2016), a atuação dos profissionais da informação no ciclo de IC (NASSIF; SANTOS, 2010), similaridades entre a IC, Gestão da Informação e Gestão do Conhecimento (CAPUANO et al., 2009), entre outros. Porém, tais estudos carecem de iniciativas que integrem as especificidades sobre a formação e atuação dos pesquisadores no desenvolvimento do corpo de conhecimento em IC, que poderiam ampliar o entendimento da IC como objeto de pesquisa da Ciência da Informação.

Com base nos desafios presentes e futuros e na importância da consolidação da atividade de IC no Brasil, este artigo tem como objetivo geral identificar e analisar o perfil do pesquisador de IC, quanto à formação, atuação e produção científica. Nesse sentido, a amostra foi composta por 1.434 currículos, dados referenciais de 223 de artigos e 26 projetos de pesquisa em andamento, recuperados de forma automatizada junto à Plataforma Lattes, com a expressão de busca "inteligência competitiva". O método de pesquisa utilizado compreendeu as técnicas de análise bibliométrica e de conteúdo. Os resultados deste artigo podem contribuir para o entendimento da interdisciplinaridade da IC e sua legitimidade como campo de pesquisa em Ciência da Informação.

\section{Aspectos da formação e atuação em inteligência competitiva}

Segundo Calof (1999) e Stefánikováa e Masárováa (2014) é difícil identificar um único foco de aplicação para a IC. Calof (1999) apresentou várias situações em que se pode fazer uso da IC no contexto organizacional. A atividade de marketing, por exemplo, utiliza a IC para monitorar rumores de novos produtos e lançamentos, para identificar as causas da organização ter perdido ou ganhado contratos, e ainda, avaliar a posição de concorrentes, além de outros. Na produção, a IC é importante para avaliar os custos comparativamente aos dos concorrentes, identificar novas tecnologias existentes e comparar tecnologias empregadas. Na área de gestão de pessoas, a IC pode ser implementada, por exemplo, para verificar se as políticas dessa área estão alinhadas com as políticas da organização como um todo, se são apropriadas para a atração e retenção de profissionais chave.

Independentemente do foco de aplicação da IC é possível representar o processo de produção de inteligência como um ciclo de atividades (Figura 1), que depende de diferentes papéis e funções de uma equipe de trabalho (CALOF, 1999; BATTAGLIA, 1999; MILLER, 2000; BOUTHILLIER; SHEREAR, 2003; NIT, 2004; LAHEY, 2003; PRESCOTT, 2009; AMARAL et al., 2011). A consolidação adequada dessa equipe é tida como essencial para que suas atividades gerem os melhores resultados, portanto, inteligência acionável. Isso ocorre devido a própria natureza da IC, que exige profissionais competentes, cuja atuação está pautada em conhecimentos, habilidades e atitudes condizentes com o trabalho que executam, geralmente dividida em papéis que representam todas as 
funções necessárias à realização da IC: coordenador, analista e coletor (LAHEY, 2003; AMARAL et al., 2011). Nessa perspectiva, a experiência e o talento são fundamentais para a prática da IC (FULD, 1995). Há várias discussões ainda não resolvidas envolvendo os profissionais de IC e a sua formação, dentre elas, se os profissionais de IC nascem ou se são formados ao longo do tempo. Tais discussões são relevantes para que se entenda as competências desses profissionais e o tipo de conhecimento, habilidade e atitude, que demonstram ao executarem efetivamente o ciclo de IC (AMARAL et al., 2011; LAHEY, 2003).

Gilad (2003) afirma que é fundamental para a formação do profissional de IC mesclar educação e treinamento, o que denominou de Education plus Training. A educação proporciona a aquisição e a compreensão de áreas da informação (conceitos e teorias) e o treinamento possibilita a aplicação em um campo, desenvolvendo a autoconfiança dos profissionais. Para Miller (1994), essencialmente há duas formas de oferta educacional em IC disponível: educação formal (graduação e pós-graduação - especialização, mestrado, doutorado) e autodidata (apresentada em maior quantidade, porém é o próprio indivíduo que planeja a sua formação). A educação formal em IC recebe muitas críticas por parte dos especialistas, afirmando que elas são deficitárias (SAWKA, 1999; CALOF, 1999; MILLER, 1994; 2000). Embora tenha havido uma evolução de ofertas disponíveis, alguns fatores chave impediram que o número de ofertas educacionais em IC crescesse rapidamente. Alguns desses fatores foram apontados por Miller (1994): poucas ofertas de cursos/programas - há uma carência generalizada de ofertas de IC no nível de pós-graduação; escassez de publicações; ambiguidade da área; tendências econômicas; integração entre cursos; ausência de faculdades qualificadas para ministrarem cursos e um conjunto de áreas que queira hospedá-la. Merriti (1999) cita três pontos necessários à expansão acadêmica: material de ensino (livros didáticos de IC), professores com formação em IC e demanda por profissionais de IC. Apesar dessas dificuldades encontradas, a IC vem ganhando aceitação dentro do contexto universitário, onde houve um pequeno desenvolvimento positivo em educação.

Diferente do que ocorreu nos Estados Unidos, Europa e Japão, a introdução da IC no Brasil não ocorreu por meio de ex-agentes de Serviços de Inteligência $e$, sim, por profissionais da Ciência da Informação. O movimento desses profissionais, além de corajoso, foi de inegável importância para a promoção e desenvolvimento da IC no Brasil (MARCIAL, 2004). Em 1996, o Instituto Nacional de Tecnologia (INT), órgão do Ministério de Ciência e Tecnologia empreendeu um conjunto de ações visando promover maior difusão da IC no país. Dentre essas ações foi celebrado um acordo de cooperação com a Université Aix-Marseille III - Centre de Recherches Retrospectives de Marseille (CRRM), da França, visando a transferência de tecnologias e metodologias desenvolvidas nas áreas de Inteligência Competitiva e Tecnológica para as instituições e empresas brasileiras. Como primeira etapa foi realizado em 1996 o curso 
de "Informação Estratégica", que contou com doze alunos, dos quais seis desenvolveram pesquisas na área, em suas teses de doutorado. $\mathrm{Na}$ segunda etapa, ocorrida no período de 1997-2002, reuniram-se competências existentes no governo, nas universidades e na indústria, parcerias nacionais e internacionais, para aprimoramento do curso (Informação Estratégica) e sua adaptação à realidade brasileira. Em 2000, - Curso de Especialização em Inteligência Competitiva (CEIC), foi realizado em cinco diferentes cidades brasileiras, resultando na formação de 110 profissionais e na disseminação dos conhecimentos de forma muito rápida. Os trabalhos elaborados pelos alunos do curso contribuíram de forma efetiva para o início da criação de uma literatura nacional sobre o assunto, praticamente inexistente (COELHO et al., 2006).

Esse curso de especialização contribuiu também para o surgimento inicial de uma rede de especialistas que conta com professores de diferentes universidades brasileiras. Foram criadas, por ex-alunos do CEIC, duas associações ligadas à IC: Associação Brasileira dos Analistas de Inteligência Competitiva (ABRAIC, 2016), fundada em 2000 e a Associação dos Ex-Alunos de Inteligência Competitiva - IC Brasil fundada em 2004. Por iniciativa de ex-alunos, com o apoio da FINEP, foi organizado o Workshop Brasileiro de Inteligência Competitiva e Gestão do Conhecimento, que está em sua décima segunda (12a) edição. Em agosto de 2006 foi realizado o I Congresso Ibero-Americano de Inteligência Competitiva e Gestão do Conhecimento, primeiro evento de alcance internacional, que viabilizou contribuições de pesquisadores ibero americanos ao Brasil (COELHO et al., 2006).

As edições do Workshop Brasileiro de Inteligência Competitiva e Gestão do Conhecimento realizados nos anos de 1999, 2002 e 2009 merecem ser destacadas. A edição realizada em 1999, no Rio de Janeiro, foi a primeira e representa o marco inicial da IC no Brasil em termos de divulgação e compartilhamento do conceito e das práticas de IC. O evento de 2002 realizado em São Paulo destaca-se pelo expressivo número de participações (632 congressistas) e pela apresentação de 132 trabalhos científicos e empresariais, indicando que a IC já se tornara um assunto de amplo interesse no país. Quanto ao evento de 2009 realizado em Manaus (AM), além da sua importância para o compartilhamento de conhecimentos e práticas, sua ocorrência na Região Norte foi um marco em termos de disseminação da IC por todas as regiões do país.

Diante da percepção da importância de se construir um corpo de conhecimento sólido em IC, algumas instituições de ensino, como a Universidade Federal de Minas Gerais (UFMG), a Universidade Federal de Santa Catarina (UFSC), o Instituto Brasileiro de Informação em Ciência e Tecnologia (IBICT) e as associações profissionais, como a Society of Competitive Intelligence Professionals (SCIP, 2016) nos Estados Unidos e a Associação Brasileira dos Analistas de Inteligência Competitiva (ABRAIC, 2016), criaram cursos e programas específicos para o aperfeiçoamento desses profissionais que atuam em um mundo em constante transformação, exigindo profissionais cada vez mais qualificados e aptos 
para oferecer respostas satisfatórias aos problemas e aspirações organizacionais (MILLER, 2002).

Após mais de 19 anos decorridos desde sua inserção no país, a IC encontra-se difundida tanto nos meios acadêmico como empresarial. Em consulta realizada à base de currículos Lattes (CNPQ, 2015a) utilizando a expressão de busca "inteligência competitiva" foram encontrados 712 doutores, de diferentes formações, associados ao tema, e, em consulta feita ao Diretório de Grupos de Pesquisa (CNPQ, 2015b), utilizando-se a mesma expressão de busca, foram encontrados 38 grupos de pesquisa associados ao tema. No meio empresarial no Brasil é possível identificar iniciativas de IC na área de energia, telecomunicações, bancária, varejo e farmacêutica, dentre outras, confirmando que IC pode ser utilizada por qualquer organização independente do setor industrial no qual esteja inserida (GOMES; BRAGA, 2006b). Menezes (2005) de forma indireta cita algumas organizações brasileiras que utilizam IC: Petrobras, Serviço Nacional de Aprendizagem Industrial (SENAI); Financiadora de Estudos e Projetos (FINEP); Empresa Brasileira de Correios e Telégrafos, Polo Calçadista de Jau-SP e Empresa Brasileira de Compressores S. A. (EMBRACO).

A profissionalização da IC passa pela formação de um corpo de conhecimento particular, onde os profissionais são vistos como experts em demonstrar competências em processos, aplicando um conjunto especializado de conhecimento, além do desenvolvimento de habilidades e atitudes. Esse conjunto único de conceitos, métodos e teorias irão compreender o "corpo de conhecimento" derivado do aprendizado acadêmico e de pesquisa cientifica. Ele deverá ser constantemente testado, ampliado e atualizado através de pesquisas. Em uma profissão o corpo de conhecimento é adquirido por uma rigorosa preparação, tipicamente através de uma graduação ou pós-graduação. Por causa da existência desse corpo de conhecimento, praticantes de uma profissão podem ter suas competências e aprendizados avaliados por intermédio de padrões derivados dele (FLEISHER, 2003). É importante ressaltar que todos os indivíduos que atuam em IC têm o dever de contribuir para o desenvolvimento desse corpo de conhecimento (KOLB, 1999). Nesse sentido, a pesquisa científica propicia a descoberta de gargalos de conhecimento, geração de questões e hipóteses, compreensão do estado da arte sobre conceitos, teorias e práticas, absorção de métodos e técnicas de pesquisa, comparação e validação de resultados empíricos, dentre outros. É uma atividade que contribui decisivamente na indagação e (re)construção da realidade almejada na pesquisa, e também alimenta o processo de ensino (LIMA; MIOTO, 2007).

Segundo Tenopir e King (2001) levantamentos mostram que artigos de periódicos são as fontes de informação mais lidas e consideradas as mais relevantes pelos cientistas. Oliveira Filho e outros (2005) indicam que as agências de pesquisa e de fomento estimulam a publicação dos resultados, colocando publicações em eventos como oportunidades para, diante de críticas e sugestões, aperfeiçoar a pesquisa, que antecede a 
publicação final da mesma. Dessa maneira pode-se entender que publicações em eventos, por antecederem as publicações finais de resultados de projetos de pesquisa, fazem parte do processo de construção do conhecimento, refletindo assim o estado da pesquisa no momento da publicação. Portanto, é possível intuir que seria factível perceber como está o desenvolvimento das pesquisas em IC no Brasil, a partir da análise das publicações em periódicos. Porém, há uma série de desafios para a recuperação das publicações em IC, consequentemente para a realização dessa percepção. Como por exemplo, a diversidade de base de dados de artigos científicos e suas limitações para representar a produção cientifica nacional (MILANEZ et al., 2014), e ainda, a multiplicidade de focos da IC (CALOF, 1999).

\section{Método e desenvolvimento da pesquisa}

O método de pesquisa utilizado foi do tipo exploratório, compreendeu uma abordagem quantitativa e qualitativa e as técnicas de análises bibliométrica e de conteúdo (BEUREN, 2008; QUONIAM et al., 2001; BARDIN, 2004). A "Plataforma Lattes" (PL) foi utilizada como fonte de informação, visto que uma das funcionalidades da PL é gerar currículos que se tornam públicos, disponibilizando à sociedade referências a outros documentos (alguns públicos e outros privados) do arquivo pessoal, ou institucional dos pesquisadores brasileiros (SILVA; SMIT, 2009). Os chamados Currículos Lattes são atualmente considerados um padrão para avaliação, representando um histórico das atividades científicas, acadêmicas e profissionais de pesquisadores cadastrados, sendo caracterizados pela livre inserção de dados. Nesta pesquisa a amostra de dados foi composta por 1.434 currículos, dados referenciais de 223 artigos e 26 projetos de pesquisa em andamento, recuperados de forma automatizada junto à Plataforma Lattes, com a expressão de busca "inteligência competitiva".

Para a identificação dos pesquisadores atuantes em IC, em 14 de maio 2015, foi acessada a interface de "busca avançada" da PL, no campo "esta expressão booleana", utilizou-se a expressão: "(inteligencia_competitiva)". Optou-se pelos seguintes filtros: a) nas bases - doutores e demais pesquisadores (mestres, graduados, estudantes, técnicos, etc.); b) na nacionalidade - brasileira e estrangeira; e c) país - todos. A partir da url gerada como resultado da busca, utilizouse um script (software) programado em Python para extrair os identificadores únicos (ID) de cada currículo recuperado e disponibiliza-los em um arquivo texto com a lista dos IDs de 2.160 pesquisadores.

A lista de IDs foi processada pelo software livre ScriptLattes (MENACHALCO; CESAR-JR, 2009) para a extração das informações dos currículos. O resultado foi a recuperação de informações relacionadas: 1] a atuação e formação dos 2.160 pesquisadores, contendo endereço profissional (organização e localização) e formação (áreas da graduação, especialização, mestrado e doutorado), entre outras; 2] a produção 
científica (14.495 referências bibliográficas de artigos completos publicados em periódicos); e 3] aos 4.768 projetos de pesquisa, contendo ano de início e encerramento, título, descrição, situação, natureza e integrantes dos projetos. É importante ressaltar que os scripts utilizados são livres e podem ser baixados em: https://bitbucket.org/vlab4u ${ }^{1}$.

A amostra analisada foi composta por 1.434 currículos referentes aos indivíduos graduados, requisito mínimo para pleitear uma pósgraduação no Brasil. Com o auxílio do software VantagePoint (2015) foi selecionado o corpus principal da pesquisa em IC, formado por dados referenciais de 223 artigos que continham o termo "inteligência" em seus títulos e versavam sobre IC. Visando identificar as temáticas investigadas, foi possível recuperar os textos completos de 173 artigos, dos quais foram extraídas suas palavras-chave. A recuperação parcial dos artigos completos pode estar relacionada a não indexação desses artigos em bases de dados. Na seleção dos projetos de pesquisa que compuseram a amostra, optou-se em utilizar os que apresentavam o status "Atual" na indicação de ano de término, totalizando 747 projetos. Em seguida, selecionou-se aqueles que continham a palavra "Inteligência" em seu título e/ou resumo, o que resultou em uma lista com 39 projetos. Após análise desses projetos foi possível considerar apenas 26 projetos de pesquisa em IC, pois, os demais versavam sobre outras temáticas relacionadas ao termo Inteligência. Os projetos em andamento foram selecionados por constituírem o corpus que evidencia os temas atuais das pesquisas em desenvolvimento e que potencialmente representam as publicações de médio prazo da área.

A análise de conteúdo (BARDIN, 2004) dos títulos presentes nos dados referenciais dos 223 artigos e 26 projetos em andamento, foi realizada para identificar e categorizar os artigos e projetos a partir das especificidades da teoria de IC, e, neste sentido foi necessário: 1) a criação de categorias - não existem normas fixas nem procedimentos padronizados, mas, acredita-se que um quadro teórico consistente pode auxiliar uma seleção inicial mais segura e relevante. Aspectos advindos de diferentes fontes e situações e que aparecem com certa regularidade são a base para o agrupamento da informação em categorias (LUDKE; ANDRÉ, 1986); e 2) a análise das categorias de IC foram determinadas a partir da análise das obras dos experts Fleisher e Blenkhorn (2003) e Fuld (1995). Os dados referenciais dos 223 artigos e 26 projetos em andamento foram analisados e classificados em categorias com base na abrangência de um único conceito, homogeneidade e exclusividade de cada categoria (Tabela 2).

Por fim, elaborou-se um conjunto de indicadores visando: 1] representar de forma gráfica o perfil de atuação e formação do pesquisador brasileiro em IC; 2] categorizar a produção científica e os projetos de pesquisa; e 3] correlacionar as temáticas investigadas nas publicações em IC.

${ }^{1}$ Acesso em: 20 fev. 2016. 


\section{Resultados}

A caracterização do pesquisador de IC no Brasil, quanto a sua formação e vinculação profissional está representada na Figura 1. É possível perceber a significativa concentração desses pesquisadores nas regiões Sudeste e Sul. Dos 1.434 pesquisadores da amostra analisada, 631 possuem endereço profissional na região Sudeste e 424 na região Sul, outros 119 no Distrito Federal, somando 82\% (1.174) do total da amostra em apenas seis estados - São Paulo, Rio de Janeiro, Santa Catarina, Rio Grande do Sul, Paraná, Minas Gerais - mais o Distrito Federal. Sendo 22\% só em São Paulo. O restante, 312 indivíduos, se distribuem pelos demais 20 estados. Os estados com as menores concentrações são: Pernambuco, Pará, Mato Grosso do Sul, Tocantins, Mato Grosso, Rondônia, Alagoas, Maranhão, Piauí, Sergipe e Roraima, juntos reúnem 102 profissionais (7\%). Foi possível constatar que apenas o Estado do Acre não se fez representado na amostra analisada, tal situação pode estar relacionada aos resultados das iniciativas das associações de profissionais que atuam em inteligência e aos eventos científicos, que visam a divulgação dos conceitos e práticas dessa atividade no Brasil, conforme relatado por Coelho et al. (2006).

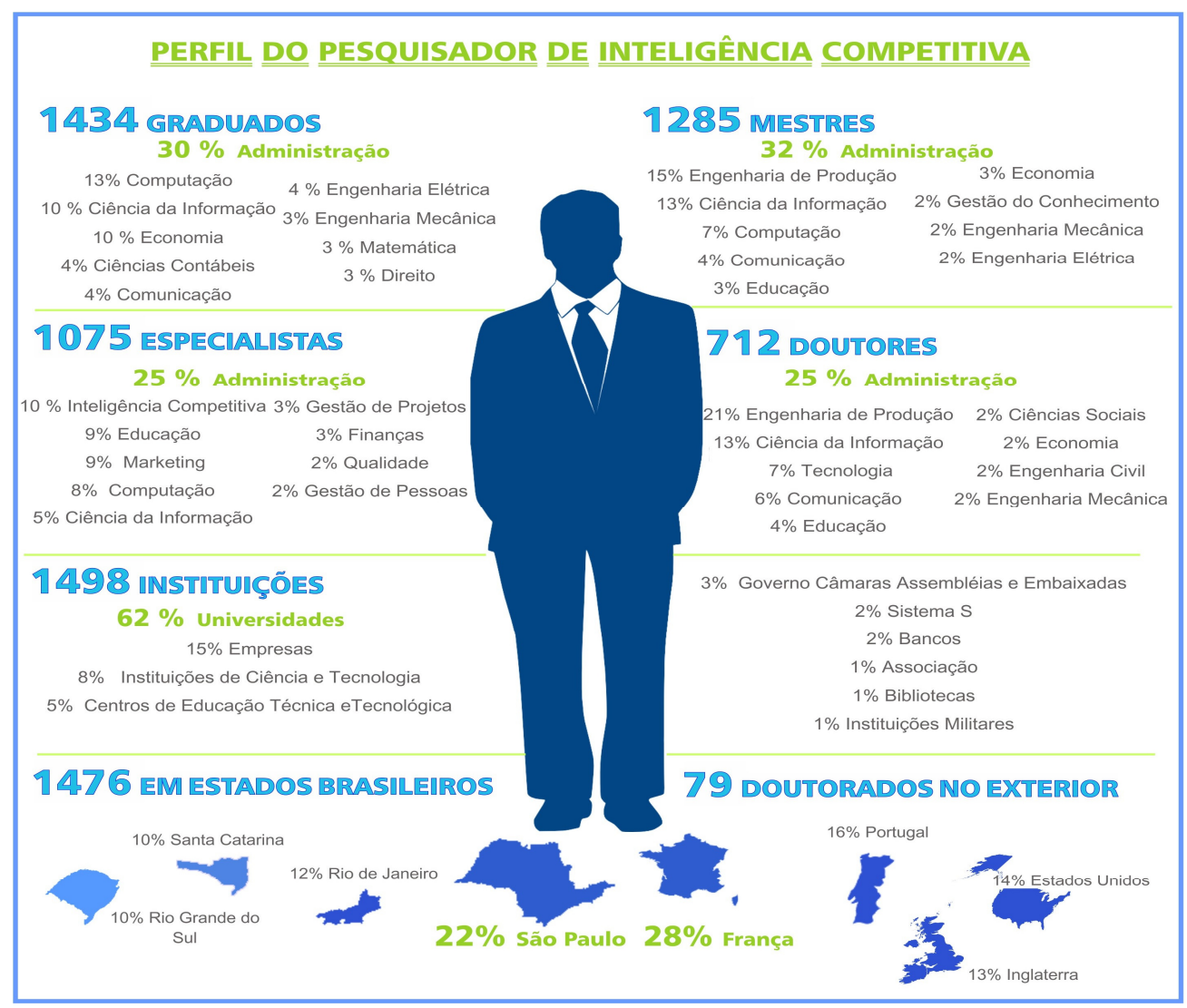


Figura 1 - Perfil do pesquisador de inteligência competitiva:

caracterização dos níveis de formação e da distribuição geográfica do profissional de IC no Brasil

Fonte: Elaborado a partir da Plataforma Lattes (CNPQ, 2015a).

Foram identificadas 1.498 organizações em que os pesquisadores de IC estão vinculados profissionalmente, sendo importante ressaltar a ocorrência de vínculo profissional em mais de uma organização. Observou-se que 928 delas, ou seja 62\%, são Universidades. As empresas somam 219 organizações, cerca de 15\%. As Instituições Científicas e Tecnológicas (ICT) seguem em terceiro lugar com 115 (8\%). Dos 238 restantes ressaltam-se aqueles vinculados a Centros de Educação (5\%), Governo (3\%), Sistema S (2\%), Bancos (1,7\%), Associações (1\%) dentre outras. No Brasil as universidades públicas têm sido o local de concentração desses pesquisadores, que têm contribuído para a expansão dos cursos de pós-graduação stricto-sensu, responsáveis pela maior parte da produção científica e seu crescimento qualitativo e quantitativo (DANTAS, 2004). Cabe ressaltar que a amostra analisada advém de uma base de dados voltada para atuações acadêmicas e científicas, porém a vinculação dos pesquisadores às empresas (15\%) pode ser explicada pelo fato de que em algum momento da sua formação se envolveram em atividades de pesquisa, como por exemplo, atividades de iniciação científica ou cursos de pós-graduação, ou receberam recursos de agências de fomento, que exigiram o cadastro do pesquisador junto a $\mathrm{PL}$, ou ainda, profissionais que atuam em parceria com a academia.

Quanto à formação, em números absolutos (Figura 1), foi possível identificar as dez áreas mais representativas nos níveis de graduação, especialização, mestrado e doutorado. Para as áreas de formação mais representativas na amostra, no nível da graduação observou-se que 30\% delas são relacionadas a Administração, sendo $13 \%$ da Computação, 10\% da Economia, 4\% das Ciências Contábeis e 4\% da Comunicação. Na formação em nível de especialização identificou-se também a área de Administração como a mais frequente com 25\%, Inteligência Competitiva $10 \%$, Educação $9 \%$, Marketing 9\%, Computação $8 \%$, Ciência da Informação 5\%, Gestão de Projetos e Finanças com 3\% cada, Qualidade e Gestão de Pessoas com 2 \% cada. Para a formação em nível de mestrado, a Administração se manteve em primeiro lugar com 32\% da amostra, Engenharia de Produção 15\%, Ciência da Informação 13\%, Computação 7\%, Comunicação 4\%, Educação 3\%, Economia 3\%, Gestão do Conhecimento, Engenharia Mecânica e Engenharia Elétrica com 2\% respectivamente. Por fim, no Doutorado a área de Administração também foi classificada com a maior concentração, 25\%, Engenharia de Produção $21 \%$, Ciência da Informação 13\%, Tecnologia 7\%, Comunicação 6\%, Educação 4\%, Ciências Sociais, Economia, Engenharia Civil e Engenharia Mecânica com 2\% respectivamente. A distribuição dos pesquisadores pelas diversas áreas do conhecimento corrobora com a afirmação de que a IC é uma disciplina interdisciplinar (MARCIAL; SUAIDEN, 2016), que pode 
estar relacionada a necessidade de manipulação e combinação de múltiplos métodos e técnicas de coleta e análise de informações, sobre a organização e seu ambiente (LAHEY, 2003), ou ainda, de que é difícil identificar um único foco de aplicação para a IC (CALOF,1999; STEFÁNIKOVÁA; MASÁROVÁA, 2014).

Entre as áreas com maior representatividade na amostra analisada, a área de Administração se manteve como a primeira colocada em todos os níveis de formação dos pesquisadores, uma vez que isto pode estar relacionado ao fato da IC ser permeada de atividades diretamente ligadas ao nível estratégico, gerencial e técnico dos processos de gestão empresarial, que revelam focos da aplicação da IC no contexto organizacional (VARGAS; SOUZA, 2001; KATZ, 1974). Ainda que de forma menos expressiva a Ciência da Informação, como área de conhecimento, também esteve presente em todos os níveis de formação. Como já apontado foi por meio de um grupo de pesquisadores franceses da Ciência da Informação, que se deram as primeiras iniciativas em IC no Brasil, podendo-se mesmo considerar que foi a área responsável pela chegada da IC no Brasil (COELHO et al., 2006). Já a necessidade de desenvolvimento de ferramentas para a automação das técnicas de coleta, tratamento e análise de informação, pode justificar a significativa frequência de pesquisadores da área de Computação. Segundo Tarapanoff (2001, p. 45) para se ter "inteligência é preciso contar com uma infraestrutura de telecomunicações como base, utilizar computadores e softwares e gerar conteúdos informacionais, em forma de base de dados, produtos e serviços". Tais ferramentas podem se basear na utilização de redes neurais e inteligência artificial para apoiar o profissional de IC, por exemplo, a prever eventos relacionados a preços, vendas e outras atividades econômicas (AIKEN, 1999). Para a área de Engenharia, quando somadas todas as variantes, foi possível observar nos níveis de mestrado (19\%) e doutorado (25\%) uma forte representatividade, ocupando a segunda posição do ranking. Tal fato pode ter relação com a importância da IC para a resolução de problemas, como por exemplo, implementação de soluções em pesquisa e desenvolvimento em situações aplicadas ao contexto empresarial, científico e tecnológico.

Visando identificar as influências internacionais no desenvolvimento da IC no Brasil, buscou-se identificar os países onde os pesquisadores doutores foram titulados. Dos 712 doutores presentes na amostra, 633 deles ou $89 \%$, são formados em Instituições do Brasil. Dentre os 79 pesquisadores doutorados no exterior, o país onde mais se formaram doutores (cadastrados na PL) atuantes em IC, foi na França com 22 doutores (28\%), em Portugal 14 (15\%), Estados Unidos 11 (14\%), Inglaterra $10(13 \%)$ dentre outros menos representativos, em números absolutos, como: Espanha 7; Argentina 4; Alemanha 2; Canadá 2; Uruguai 2; Bélgica 1; e Finlândia 1 . O relacionamento internacional com a França provavelmente deve-se ao grupo francês em Ciência da Informação que liderou a disseminação da IC no Brasil. Tal fato pode estar relacionado a origem da IC no Brasil, conforme indicado pela ABRAIC 
(2016) e Coelho e colaboradores (2006), o que caracterizou uma atuação brasileira mais voltada à influência europeia, envolvendo análises com maior ênfase em informações científicas e tecnológicas.

Ainda sobre a formação dos pesquisadores de IC, com base nas três áreas do conhecimento mais representativas na amostra analisada, os valores absolutos sobre a titulação de mestrado e doutorado realizados no Brasil, foram normalizados com base no número de PPG ativos (CNPQ, 2016b) e no número total de pesquisadores titulados (mestrado 19962009, doutorado 1996-2008) pelos PPG stricto senso aprovados pela CAPES (2016a) (CGEE, 2010; 2012). Com isso, foi possível elaborar dois indicadores: 1] média de pesquisadores por PPG ativos; e 2] percentual de pesquisadores titulados no período de 1996 a 2016. Conforme é possível visualizar na Tabela 1 , em relação a área de Administração e Engenharia de Produção a Ciência da Informação apresenta a menor quantidade de PPG ativos no Brasil, 17 de mestrado e 12 de doutorado, porém apresentou as maiores médias de titulação dos pesquisadores que atuam em IC: 9,8 para o mestrado e 7,4 para o doutorado. Em relação ao percentual de pesquisadores titulados nas três áreas mais representativas que atuam em IC no Brasil, a Ciência da Informação destacou-se pelos valores alcançados, quase que metade $(46,4 \%)$ dos pesquisadores doutores em Ciência da Informação atuam em IC. Com base nos indicadores da Tabela 1, é possível afirmar, que a Ciência da Informação tem contribuído significativamente para o desenvolvimento da IC no Brasil, e ainda, que a IC é legítima como objeto de pesquisa da Ciência da Informação, com características interdisciplinares, corroborando com as afirmações de Coelho e colaboradores (2006), MARCIAL e SUAIDEN (2016).

Tabela 1 - Áreas do conhecimento mais representativas na atuação dos pesquisadores em IC

\begin{tabular}{|c|c|c|c|c|}
\hline & Titulação & Administração & $\begin{array}{c}\text { Engenharia de } \\
\text { Produção }\end{array}$ & $\begin{array}{l}\text { Ciência da } \\
\text { Informação }\end{array}$ \\
\hline \multirow[t]{2}{*}{ Formação } & Mestrado & 4411 & 192 & 167 \\
\hline & Doutorado & 178 & 146 & 89 \\
\hline \multirow{2}{*}{$\begin{array}{c}\text { Número de PPG } \\
\text { Ativos }\end{array}$} & Mestrado (acadêmico) & 76 & 37 & 17 \\
\hline & Doutorado & 39 & 22 & 12 \\
\hline \multirow{2}{*}{$\begin{array}{l}\text { Média de pesquisadores por } \\
\text { PPG ativos }\end{array}$} & Mestrado & 5,4 & 5,2 & 9,8 \\
\hline & Doutorado & 4,6 & 6,6 & 7,4 \\
\hline \multirow{2}{*}{$\begin{array}{c}\text { Número de pesquisadores } \\
\text { titulados pelos PPG } \\
\text { brasileiros }\end{array}$} & Mestrado (acadêmico) & 15603 & 7538 & 1101 \\
\hline & Doutorado & 1029 & 1130 & 192 \\
\hline \multirow{2}{*}{$\begin{array}{c}\text { Percentual dos } \\
\text { pesquisadores que atuam em } \\
\text { IC no Brasil }\end{array}$} & Mestrado (acadêmico) & $2,6 \%$ & $2,5 \%$ & $15,2 \%$ \\
\hline & Doutorado & $17,3 \%$ & $12,9 \%$ & $46,4 \%$ \\
\hline
\end{tabular}

Fonte: Elaborada com base na amostra de 1.434 pesquisadores e nas informações disponibilizadas na SUCUPIRA (CAPES, 2016b) e CGEE (2010; 2012).

Por intermédio da elaboração e aplicação de uma categorização (SÁSILVA; ALMEIDA; GUINDANI, 2009), baseada na análise da bibliografia 
especializada da área de IC (FLEISHER; BLENKHORN, 2003; FULD, 1995), a amostra analisada sobre a produção cientifica de IC no Brasil, composta pelos dados referenciais de 223 artigos e 26 projetos, foi categorizada em: 1] Processo de Inteligência Competitiva, 2] Inteligência Competitiva impacto, 3] Teoria da Inteligência Competitiva, 4] Ferramentas, 5] Fontes de Informação, 6] Técnicas de Análise; 7] Profissional; e 8] Publicações. A partir da análise dos resultados, externalizados pela Tabela 2, pode-se observar a descrição de cada uma das categorias utilizadas neste artigo e o número de referências classificadas. As iniciativas brasileiras estão concentradas em questões voltadas à descrição de como se deve executar - processo de IC com $34,6 \%$ dos projetos em andamento nas universidades brasileiras e $30,5 \%$ dos artigos. Em segundo lugar nota-se que ainda há necessidade de afirmação da área, pois 7,7\% dos projetos e $23,8 \%$ dos artigos buscam afirmar a importância e as necessidades do desenvolvimento de IC para a competitividade das organizações. Nesse sentido, vale ressaltar que $14,3 \%$ dos projetos e $15,4 \%$ dos artigos discutem, exclusivamente, conceitos e teorias de IC. A distribuição das iniciativas nas categorias analisadas indica um estágio de desenvolvimento embrionário da IC no Brasil, em que os profissionais brasileiros buscam discutir e internalizar seus conceitos.

Tabela 2 - Descrição das categorias e distribuição dos artigos e projetos em IC

\begin{tabular}{llcc}
\hline \multicolumn{1}{c}{ CATEGORIA } & \multicolumn{1}{c}{ DESCRIÇÃo } & No ARTIGOS & No PROJETOS \\
\hline $\begin{array}{l}\text { 1] Processos de Inteligência } \\
\text { Competitiva }\end{array}$ & Descrição, elaboração e sugestão de processos e ciclos de IC. & 68 & 9 \\
\hline $\begin{array}{l}\text { 2] Inteligência Competitiva - } \\
\text { impacto }\end{array}$ & $\begin{array}{l}\text { Ressaltam a relevância e dificuldades da aplicação de IC, assim como } \\
\text { suas contribuições, GAPS e avaliação de eficiência. }\end{array}$ & 53 & 2 \\
\hline $\begin{array}{l}\text { 3] Teoria da Inteligência } \\
\text { Competitiva }\end{array}$ & $\begin{array}{l}\text { Conceituação e definição de termos relacionados a IC (revisão } \\
\text { teórica). }\end{array}$ & 32 & 4 \\
\hline 4] Ferramentas & Instrumentos e softwares utilizados no desenvolvimento de IC. & 22 & 6 \\
\hline 5] Fontes de Informação & Recursos que atendam a demanda de informação para IC. & 17 \\
\hline 6] Técnicas de Análise & $\begin{array}{l}\text { Define métodos para análise das informações para desenvolvimento } \\
\text { de IC }\end{array}$ & 14 & 2 \\
\hline 7] Profissional & $\begin{array}{l}\text { Formação, qualificação e características dos IC, e em qual parte do } \\
\text { processo de IC o profissional está apto a atuar. }\end{array}$ & 14 \\
\hline 8] Publicações & $\begin{array}{l}\text { Análise quantitativa da produção bibliográfica na área de IC, por } \\
\text { exemplo, bibliometria. }\end{array}$ & 3 \\
\hline
\end{tabular}

Fonte: Elaborado a partir de Lattes (CNPQ, 2015a). 
A partir dos resultados é possível perceber um considerável interesse em Fontes de Informação e Ferramentas para realização da IC quanto aos artigos e projetos que versam sobre os profissionais que atuam em IC e suas formações nota-se que, quase que exclusivamente, relatam as atuações do Cientista da Informação ou Bibliotecário e as competências necessárias aos profissionais de IC. Embora tenha ocorrido a necessidade da criação da categoria "Publicações", há pouquíssimas produções que tratam do assunto, apenas $1 \%$ dos artigos buscam realizar uma análise quantitativa das publicações em IC.

As palavras-chave presentes nos artigos científicos são utilizadas para a elaboração de ranking sobre as principais temáticas e tendências de desenvolvimento em um campo científico (QUONIAM et al., 2001). A Figura 2 representa a correlação e a intensidade dos conceitos em IC investigados no Brasil, por intermédio da análise de 245 palavras-chave extraídas do texto completo de 173 artigos. Essa variedade de palavraschave, pode estar relacionada a diversidade de focos da IC (CALOF, 1999) e da pluralidade de competências necessárias ao profissional de IC (FLEISHER, 2003; LAHEY, 2003; PRESCOTT, 2009; AMARAL et al., 2011), ou ainda, à interdisciplinaridade da IC (MARCIAL; SUAIDEN, 2016).

A moldura analítica aplicada à Figura 2 compreende as seguintes observações: o tamanho das palavras-chave representa o número de artigos em que a palavra-chave ocorre na amostra analisada, sua proximidade se dá pela intensidade da co-ocorrência das palavras-chave no artigo, o algoritmo estatístico Force Atlas 2 do software Gephi, tende a aproximar os nós (palavras-chave) que apresentam maior co-ocorrência e afastar o contrário. As linhas representam as ligações (a co-ocorrência) entre as palavras-chave na amostra analisada (173 artigos). É importante ressaltar que a palavra-chave IC foi omitida para facilitar o processo de visualização, mas ela representa o centro da Figura 2. 


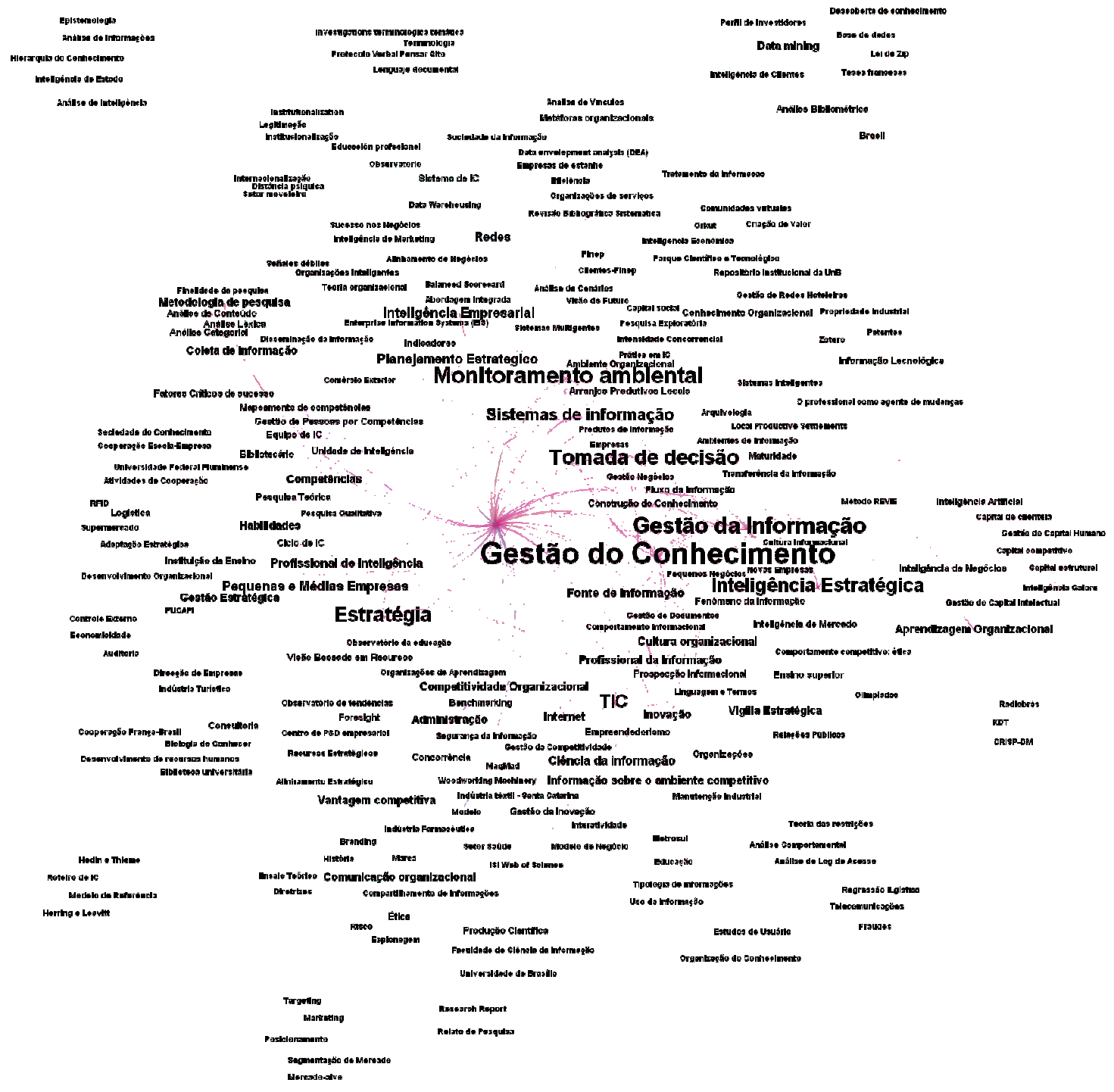

Figura 2 - Palavras-chave utilizadas pelos 173 artigos de IC e suas correlações

Fonte: Dados da pesquisa.

Com base nessa moldura analítica é possível visualizar na Figura 2 as principais temáticas correlacionadas com a IC, evidenciando uma forte aproximação com:

a) Gestão do Conhecimento (27 artigos - 12,3\%), Gestão da Informação (19 artigos - 8,7\%) e Sistemas de Informação (11 artigos - 5\%) - é possível compreender essa significativa relação com a IC, pois se o conhecimento está na cabeça das pessoas, se faz necessário implementar iniciativas em gestão do conhecimento no contexto organizacional visando viabilizar um ambiente eficiente e eficaz para o acesso e o compartilhamento de informação e conhecimento entre os 
trabalhadores (DAVENPORT; PRUSAK, 1998; STEFÁNIKOVÁA; MASÁROVÁA, 2014);

b) Monitoramento ambiental (18 artigos - 8,2\%); Estratégia (16 artigos - 7,3); Tomada de Decisão (16 artigos - 7,3\%); e Inteligência estratégica (15 artigos - 6,8\%): Essas temáticas representam a essência da atividade de inteligência, que compreende a análise das informações do contexto competitivo das organizações, visando apoiar o processo de tomada de decisão, fornecendo inteligência acionável aos decisores (FULD, 1995);

c) TIC - Tecnologia da Informação e Comunicação (13 artigos - 5,9 \%): As TIC a partir da década de 80 impactaram diretamente a atividade de inteligência, tanto na sistematização de técnicas de análise como na maximização da capacidade de produção, armazenamento, recuperação e tratamento de significativos volumes de informações a respeito do ambiente competitivo (TARAPANOFF, 2001).

Quanto à denominação aplicada à atividade de IC, foi possível constatar que a palavra-chave IC, omitida na Figura 2, mas representada pelo seu centro, foi a mais representativa, presente em 139 artigos (64\%) da amostra analisada. É importante ressaltar que a amostra foi selecionada levando-se em conta a presença da palavra-chave "inteligência" no título dos artigos. Autores como Brody (2008) e Carvalho (2001) afirmam que há uma diversidade de termos sendo utilizados na denominação da atividade de inteligência, o que corrobora com os resultados encontrados nesta pesquisa, entre os termos mais utilizados pelos pesquisadores da área estão: 1] Inteligência Estratégica (15 artigos - 6,8\%) e 2] Inteligência Empresarial (07 artigos - 3,2\%) - também é possível identificar outros termos com menor intensidade de frequência nos artigos, como por exemplo, Inteligência de Mercado, Inteligência de Negócios, Inteligência Econômica, Inteligência de Clientes, Inteligência de Estado e Inteligência de Marketing. Devido a diversidade de focos da IC e a variedade de termos utilizados pelos pesquisadores, recomenda-se, para trabalhos futuros em IC, que se leve em consideração outros termos adicionais, visando aumentar a revocação dos documentos (currículos) que poderão compor as futuras amostras, o que contribuiria para uma maior abrangência dos estudos relacionados a IC, em especial no contexto brasileiro.

Por intermédio da frequência das palavras-chave foi possível visualizar as técnicas de análise de informações mais utilizadas pelos profissionais de IC nessa amostra: Data mining, Análise bibliométrica, Análise Categorial, Análise de Conteúdo, Análise Léxica, Benchmarking, Fatores Críticos de sucesso, Foresight, Prospecção Informacional, Análise Comportamental, Análise de Cenários, Análise de Informações, Análise de Log de Acesso, Análise de Vínculos, Balanced Scorecard, Data 
envelopment analysis (DEA), Data Warehousing e Estudos de Usuário. É importante ressaltar a ausência de técnicas de análise como, SWOT e Cinco Forças de Porter, que segundo Fleisher e Bensoussan (2002), são as técnicas de análise de informações mais utilizadas pelos profissionais de IC, devido a sua facilidade de compreensão e aplicação. Essa ausência pode estar relacionada ao perfil da amostra analisada, composta em sua maioria por pesquisadores da academia brasileira.

\section{Conclusão}

O presente artigo abordou as especificidades sobre a formação e atuação dos pesquisadores brasileiros no desenvolvimento do corpo de conhecimento em IC, por intermédio da análise das informações a respeito das iniciativas desses pesquisadores registradas na PL.

Os resultados alcançados possibilitaram a identificação e análise do perfil do pesquisador, da produção científica e dos projetos em IC no Brasil, e ainda, a identificação das temáticas com maior intensidade de iniciativas e os conceitos correlacionados com a IC. Os resultados alcançados poderão ser utilizados por instituições de pesquisa ou profissionais que desejem aprofundar seus conhecimentos em IC, uma vez que fornece insights sobre o perfil do pesquisador e aponta as iniciativas de pesquisa e seus resultados no desenvolvimento do corpo de conhecimento em IC no país.

A partir da análise dos resultados observou-se que os pesquisadores de IC em sua maioria estão vinculados às universidades públicas, concentrados nas regiões Sudeste e Sul. Os pesquisadores de IC apresentam alto nível de formação acadêmica e influências internacionais, em especial dos pesquisadores franceses da Ciência da Informação. Entre as áreas de conhecimento relacionadas à titulação de mestrado e doutorado, a Ciência da Informação se destacou pelos números alcançados, absolutos e normalizados, em relação aos pesquisadores titulados que atuam no desenvolvimento do corpo de conhecimentos da IC. E ainda, que essa atuação, pode ser ampliada com a criação de novos PPG em Ciência da Informação no Brasil. Assim, como a titulação (mestrado, doutorado) dos pesquisadores o corpo de conhecimento brasileiro em IC apresenta características interdisciplinares e áreas correlatas como Administração, Engenharia, Computação entre outras.

O método de pesquisa utilizado pode ser replicado para outras áreas de conhecimento, pois compreende uma sistemática para a identificação, acompanhamento e análise da produção científica no Brasil, por intermédio das informações coletadas junto à PL. Sua vantagem está na automação da coleta e tratamento das informações registradas na PL. É importante ressaltar que este artigo focou nas formações dos pesquisadores de IC, nas publicações cientificas em periódicos e nos projetos de pesquisa em andamento, mas que a análise das informações registradas na $\mathrm{PL}$ possibilitam outros resultados. Por exemplo, com base na análise das informações referentes aos produtos tecnológicos, poderia 
ser possível identificar iniciativas de sistematização da IC na forma de ferramentas, como por exemplo, softwares utilizados para automatizar técnicas de coleta e análise de informações.

Conclui-se com base no referencial teórico e nos resultados alcançados que a produção científica brasileira está concentrada no desenvolvimento teórico da inteligência competitiva, abordando de forma incipiente casos ilustrativos sobre a sua aplicação. Com relação ao profissional atuante em IC constatou-se o alto nível de formação formal com 712 doutores e uma grande concentração desses indivíduos na região Sudeste e Sul e no ambiente das universidades.

\section{Referências}

$\begin{array}{rrrr}\text { ASSOCIAÇÃO } & \text { BRASILEIRA DOS } & \text { ANALISTAS EM } & \text { INTELIGÊNCIA } \\ \text { COMPETITIVA } & \text { (ABRAIC). } & \text { Disponível } & \text { em: }\end{array}$
<http://www.abraic.org.br/index.php?idAtual=1>. Acesso em: 10 out. 2016.

AIKEN, M. Competitive intelligence through neural networks. Competitive Intelligence Review, v. 10, n. 1, p. 49-53, 1999.

AMARAL, R. M. et al. Perfis de competências relativas à inteligência competitiva: um estudo exploratório no Brasil. Ciência da Informação, v. 40, n. 2, p. 125-144, maio/ago. 2011.

BARDIN, L. Análise de conteúdo. 3. ed. Lisboa: Edições 70, 2004.

BATTAGLIA, M. G. B. A Inteligência competitiva modelando o Sistema de Informação de Clientes - Finep. Ciência da Informação, Brasília, v. 28, n. 2, p. 200-214, maio 1999.1 Disponível em: <http://www.scielo.br/scielo.php?script=sci_arttext\&pid=S0100$19651999000200012 \& \mathrm{lng}=\mathrm{en} \& \mathrm{nrm}=$ iso $>$. Acesso em: $16 \mathrm{dez} .2015$.

BEUREN, I. M. Metodologia da pesquisa aplicável às ciências sociais. São Paulo: Atlas, 2008.

BOUTHILLIER, F.; SHEREAR, K. Assessing competitive intelligence software: a guide to evaluating CI technology. New Jersey: Information Today, 2003. 187 p.

BRODY, R. Issues in defining competitive intelligence: an exploration. Journal of Competitive Intelligence and Management, v. 4, n. 3, p. 3-16, 2008.

CALOF, J. L. Teaching CI: opportunities and needs. Competitive Intelligence Magazine, v. 2, n. 4, p. 28-31, Oct./Dec. 1999.

COORDENAÇÃO DE APERFEIÇOAMENTO DE PESSOAL DE NÍVEL SUPERIOR (CAPES). GeoCapes. 2016a. disponível em: <http://www.capes.gov.br/component/content/article/91-conteudoestatico/avaliacao-capes/6886-geocapes>. Acesso em: 30 jun. 2016. 
COORDENAÇÃO DE APERFEIÇOAMENTO DE PESSOAL DE NÍVEL SUPERIOR (CAPES). Sucupira. 2016b. Disponível em: < https://sucupira.capes.gov.br/sucupira/>. Acesso em: 30 jun. 2016.

CAPUANO, E. A. et al. Inteligência competitiva e suas conexões epistemológicas com gestão da informação e do conhecimento. Ciência da Informação, Brasília , v. 38, n. 2, p. 19-34, ago. 2009. Disponível em: $<$ http://www.scielo.br/scielo.php?script=sci_arttext\&pid=S0100$19652009000200002 \&$ Ing=en\&nrm=iso >. Acesso em: 23 jun. 2016.

CARVALHO, K. Disseminação da informação e informação de inteligência organizacional. Datagramazero, v. 2, n. 3, p. 35-46, jun. 2001.

CENTRO DE GESTÃO E ESTUDOS ESTRATÉGICOS (CGEE). Doutores 2010: estudos da demografia da base técnico-científica brasileira. Brasília, DF: Centro de Gestão e Estudos Estratégicos, 2010, 508 p.

CENTRO DE GESTÃO E ESTUDOS ESTRATÉGICOS (CGEE). Mestres 2012: Estudos da demografia da base técnico-científica brasileira. Brasília, DF: Centro de Gestão e Estudos Estratégicos, 2012. 428 p.

COELHO, G. M. et al. Ensino e pesquisa no campo da inteligência competitiva no Brasil e a cooperação franco-brasileira. Puzzle, n. 23, ano 6, 12-19, ago./out. 2006.

CONSELHO NACIONAL DE DESENVOLVIMENTO CIENTÍFICO E TECNOLÓGICO (CNPq). Plataforma Lattes. Disponível em: <http://lattes.cnpq.br/>. Acesso em: 22 maio 2015a.

CONSELHO NACIONAL DE DESENVOLVIMENTO CIENTÍFICO E TECNOLÓGICO (CNPq). Diretório de grupos de pesquisa. Disponível em: $<$ http://lattes.cnpq.br/web/dgp/home>. Acesso em: 12 dez. 2015b.

DANTAS, F. Responsabilidade social e pós-graduação no Brasil: idéias para (avali) ação. Revista Brasileira de Pós-Graduação, v. 1, n. 2, p. 160-172, 2004.

DAVENPORT, T. H.; PRUSAK, L. Conhecimento empresarial: como as organizações gerenciam seu capital intelectual. Rio de Janeiro: Campus, 1998.

DILWORTH, G. Are there linkages between theories of intelligence and the practice of competitive intelligence? In: FLEISHER, C. S.; BLENKHORN, D. L. Controversies in competitive intelligence: the enduring issues. Westport: Praeger, 2003. p. 243-256.

FLEISHER, C. S.; BENSOUSSAN, B. E. (Eds.). Strategic and competitive analysis: methods and techniques for analyzing business. Upper Saddle River, NJ: Prentice Hall; 2002.

FLEISHER, C. S. Are competitive intelligence practitioners professionals? In: FLEISHER, C. S.; BLENKHORN, D. L. Controversies in competitive intelligence: the enduring issues. Westport: Praeger, 2003. p. 29-44. 
FLEISHER, C. S.; BLENKHORN, D. L. Controversies in competitive intelligence: the enduring issues. Westport: Praeger, 2003.

FLEISHER, C. S. Competitive intelligence education: competencies, sources, and trends. The information Management Journal, v. 38, n. 2, p. 56-62, Mar./Apr. 2004.

FULD, L. M. The new competitor intelligence: the complete resource for finding, analyzing, and using information about your competitors. New York: John Wiley \& Sons, 1995.

GEPHI. Gephi: makes graphs handy. Disponível em: <http://gephi.github.io/>. Acesso em: 31 ago. 2015.

GILAD, B. CI education: Harvard style. Competitive Intelligence Magazine, v. 6, n. 4, p. 12-16, July.-Aug. 2003.

GOMES, E.; BRAGA, F. Construção de um sistema de inteligência competitiva. In: STAREC, C.; GOMES, E.; BEZERRA, J. (Org.). Gestão estratégica de informação e inteligência competitiva. São Paulo: Saraiva, 2006a. p. 111-123.

GOMES, E. B. P.; BRAGA, F. R. Inteligência competitiva no Brasil: uma realidade corporativa. PUZZLE, v. 6, n. 23, p. 5-10, ago./out. 2006b.

HAMEL, G.; PRAHALAD, C. K. The core competence of the corporation. Havard Business Review, v. 68, n. 3, p. 79-91, May-June 1990.

HERRING, J. P. World-class intelligence programs. Competitive Intelligence Magazine, Washington, v. 9, n. 3, p. 20-25, May./Jun. 2006.

KATZ, R. L. Skills of an effective administrator. Harvard Business Review, n. 52, p. 90-102, Sept./Oct. 1974.

KOLB, G. Educated professionals. Competitive Intelligence Magazine, v. 2, n. 4, p. 28-31, Oct./Dec. 1999.

LAHEY, R. What types of people perform competitive intelligence best? In: FLEISHER, C. S.; BLENKHORN, D. L. Controversies in competitive intelligence: the enduring issues. Westport: Praeger, 2003. p. 243-256.

LIMA, T. C. S.; MIOTO, R. C. T. Methodological procedures in the construction of scientific knowledge: bibliographic research. Revista Katálysis, Florianópolis, v. 10, n. special, p. 37-45, 2007.

LUDKE, M.; ANDRE, M. E. D. A. A pesquisa em educação: abordagens qualitativas. São Paulo: EPU, 1986.

MARCIAL, E. C. (Org.). Estudos de futuro: cenários sobre o futuro da inteligência competitiva no Brasil. Brasília: ABRAIC 2004.

MARCIAL, E. C.; SUAIDEN, E. J. A estrutura científica da inteligência competitiva. Transinformação, Campinas, v. 28, n. 1, p. 97-106, Abr. 2016. 
<http://www.scielo.br/scielo.php?script=sci_arttext\&pid=S0103-

37862016000100097\&lng=en\&nrm=iso >. Acesso em: 6 jul. 2016.

MENA-CHALCO, J. P.; CESAR-JR, R. M. Scriptlattes: an open-source knowledge extraction sytem from the Lattes Plataform. Journal of the Brazilian Computer Society, v. 15, n. 4, p. 31-39, 2009.

MENEZES, E. M. Inteligência competitiva: uma revisão de literatura. Revista Digital de Biblioteconomia \& Ciência da Informação, v. 3, n. 1, p. 103-130, jul./dez. 2005.

MERRIT, C. CI and higher education dilemma. Competitive Intelligence Magazine, v. 2, n. 4, p. 19-31, Oct./Dec. 1999.

MILANEZ, D. H. et al. Análise de bases de dados e termos de busca para estudos bibliométricos e monitoramento científico em nanocelulose. Em Questão, v. 20, p. 114-133, 2014.

MUELLER, S. P. M.; MIRANDA, A.; SUAIDEN, E. J. A pesquisa em Ciência da Informação no Brasil: análise dos trabalhos apresentados no IV Enancib, 2000. Revista de Biblioteconomia de Brasília, v.23/24, n. 3 esp., p. 293-308, 1999/2000.

MILLER, J. P. O nascimento e o crescimento do seu processo de inteligência: fatores comportamentais, culturais e estruturais. In: MILLER, J. P. (Org.). O milênio da inteligência competitiva. São Paulo: Bookman, 2002. p. 53-65.

MILLER, J. P. Skills and training for intelligence. In: FULD, L. M.; MILLER, J. P. Millennium intelligence: understanding and conducting competitive intelligence in the digital age. New Jersey: Cyber Age Books, 2000. p. 5566.

MILLER, J. P. Educational programs for intelligence professionals. Library Trends, v. 43, n. 2, p. 253-263, 1994.

NASSIF, M. Er.; SANTOS, E. L. O profissional da informação em atividades de inteligência competitiva. Informação \& Informação, [S.I.], v. 14, n. 2, p. 21-37, dez. 2010. Disponível em: <http://www.uel.br/revistas/wrevojs246/index.php/informacao/article/vie w/2477/4145 >. Acesso em: 23 Jun. 2016.

NÚCLEO DE INFORMAÇÃO TECNOLÓGICA EM MATERIAIS (NIT). Manual de inteligência competitiva. São Carlos, SP, 2004. 80f. (publicação interna).

OLIVEIRA FILHO, R. S. et al. Fomento à publicação científica e proteção do conhecimento científico. Acta Cirúrgica Brasileira, v. 20, n. 2 (suplemento), p. 35-39, jan. 2005.

PERUCCHI, V.; ARAUJO JUNIOR, R. H. de. Produção científica sobre inteligência competitiva da Faculdade de Ciência da Informação da Universidade de Brasília. Perspect. ciênc. inf., Belo Horizonte, v. 17, n. 2, p. 37-56, jun. 2012. Available from 
<http://www.scielo.br/scielo.php?script=sci_arttext\&pid=S1413-

99362012000200004\&lng=en\&nrm=iso>. Acesso em: 23 jun. 2016.

PINHEIRO, L. V. R. Ciência da Informação: desdobramentos disciplinares, interdisciplinares e transdisciplinares. In: GONZÁLEZ DE GÓMEZ, $M$. N.; ORRICO, E. G. D. (Org.). Políticas de memória e informação: reflexos na organização do conhecimento. Natal: Ed. UFRN, 2006. p. 111141.

PORTER, M. Vantagem competitiva: criando e sustentando um desempenho superior. Rio de Janeiro: Campus, 1989.

PRESCOTT, J. The CI professionals'competency framework. Magazine Competitive Intelligence, v. 12, n. 1, p. 23-26, 2009.

QUONIAM, L. et al. Intelligence obtained with the application of data mining analysing the French DocThéses on subjects about Brazil. Ciência da Informação, v. 30, n. 2, p. 20-28, May./Aug. 2001.

SOCIETY OF COMPETITIVE INTELLIGENCE PROFESSIONALS (SCIP). 2016. Disponível em: <http://www.scip.org>. Acesso em: 12 dez. 2015.

SÁ-SILVA, J. R.; ALMEIDA, C. D.; GUINDANI, J. F. Pesquisa documental: pistas teóricas e metodológicas. Revista Brasileira de História \& Ciências Sociais, v. 1, n. 1, julho 2009.

SAWKA, K. The analyst's corner: training intelligence analysts. Competitive Intelligence Magazine, v. 2, n. 2, Apr./June 1999.

SILVA, F. M.; SMIT, J. W. Organização da informação em sistemas eletrônicos abertos de informação científica e tecnológica: análise da Plataforma Lattes. Perspectivas em Ciência da Informação [on line], v. 14, n. 1, p. 77-98, 2009.

SILVA, E. L. C. et al. Panorama da pesquisa em ciência da informação no brasil. Informação \& Sociedade: Estudos, v. 16, n. 1, p. 159-177, 2006. Disponível em: <http://www.brapci.ufpr.br/brapci/v/4171>. Acesso em: 23 jul. 2006.

STEFÁNIKOVÁA, L; MASÁROVAA, G. The need of complex competitive intelligence. Procedia - Social and Behavioral Sciences, n. 110, p. 669$677,2014$.

TARAPANOFF, K. (Org.). Inteligência organizacional e competitiva. Brasília: Editora da UnB, 2001. 344 p.

TENOPIR, C.; KING, D. W. A importância dos periódicos para o trabalho científico. Revista de Biblioteconomia de Brasília, v. 25, n. 1, p. 15-26, 2001.

VANTAGEPOINT. About the VantagePoint. 2001. Disponível em: <http://www.thevantagepoint.com/>. Acesso em: 8 ago. 2015. 
VARGAS, L. M; SOUZA, R. F. O ator de inteligência competitiva (IC) nas empresas: habilidades profissionais e exigências do mercado. REAd, v. 7, n. 6, ed. Especial, nov./dez. 2001.

roniberto@ufscar.br

ligrasiele@yahoo.com.br

karingomesf@gmail.com

leandro@nit.ufscar.br 